\title{
Flexibel in Zeit und Raum - Gelingensbedingungen von Homeoffice und mobiler Arbeit in KMU
}

\author{
Jennifer Kaczynska und Angelika Kümmerling
}

\section{Einleitung}

Spätestens mit der durch die COVID-19 Pandemie kurzfristig ausgelösten Veränderungen der Arbeitswelt sind die Themen Homeoffice beziehungsweise mobiles Arbeiten endgültig im öffentlichen Bewusstsein angekommen. Bedingt durch die Möglichkeiten und zunehmende Verwendung der digitalen Informations- und Kommunikationstechnologien und verstärkt durch ein größeres Interesse an Themen wie der Work-Life-Balance oder der Vereinbarkeit von Beruf und Familie steht ortsflexibles Arbeiten bereits seit einigen Jahren auf der Agenda sowohl der Arbeitnehmer- als auch der Arbeitgeberseite. Trotz einer grundsätzlich positiven Einschätzung beider Seiten (Bonin et al. 2020), konnte sich mobiles Arbeiten oder das Arbeiten im Homeoffice jedoch vor dem Ausbruch der COVID-19 Pandemie nicht durchsetzen. So gab in Deutschland im Jahr 2019 nicht einmal jeder zehnte abhängig Beschäftigte an, in den letzten vier Wochen zumindest einmal von zu Hause gearbeitet zu haben; dies ist im europäischen Vergleich ein unterdurchschnittlicher Wert (Eurostat 2020). Dabei variiert die Möglichkeit mobil oder im Homeoffice zu arbeiten stark zwischen Beschäftigtengruppen und unterscheidet sich nach Betriebsmerkmalen und Branchenzugehörigkeit: Beschäftigte in Dienstleistungsbranchen arbeiten häufiger im Homeoffice als Beschäftigte des Verarbeitenden Gewerbes, Höherqualifizierte und insbesondere Beschäftigte mit Führungsverantwortung wiederum häufiger als geringer Qualifizierte und Arbeitnehmer*innen ohne Führungsposition. Beschäftigte mit kognitiven Tätigkeiten haben häufiger Zugang zu Homeoffice als diejenigen, die überwiegend mit manuellen Tätigkeiten betraut sind. Und nicht zuletzt ist das betriebsunabhängige Arbeiten in Großunternehmen verbreiteter als in kleinen und mittleren Unternehmen (KMU) - wobei eine neuere Analyse von Mergener (2020) zeigt, dass der Einfluss der Betriebsgröße quer zum Einfluss der Tätigkeiten liegt (siehe auch Brenke 2016). Das heißt, auch bei gleicher Tätigkeit ist die Wahrscheinlichkeit, diese (auch) arbeitsstättenfern ausüben zu können, in Großunternehmen höher als in KMU. Dieser Befund bildet 
den Ausgangspunkt unseres Beitrags, in dem wir fragen, mit welchen Herausforderungen KMU hinsichtlich der Einführung von ortsflexiblem Arbeiten konfrontiert sind und wie sie diesen begegnen. Ziel ist es herauszuarbeiten, welche Bedingungen gegeben sein müssen, damit Homeoffice und mobile Arbeit auch in KMU gelingen können. „Gelingen“ bedeutet in unserem Fall nicht nur Ermöglichung und Realisierung unter Beachtung gesetzlicher Bestimmungen wie etwa dem Arbeitsschutz, sondern hat durchaus auch eine wertende Komponente und impliziert Zufriedenheit mit der Ausgestaltung und Umsetzung des Homeoffice. Dabei müssen wir allerdings einschränkend feststellen, dass wir uns im vorliegenden Beitrag vor allem auf die Sichtweise und Einschätzungen der Geschäftsführungen konzentrieren. Die Zufriedenheit der Beschäftigten wird daher nur sehr vermittelt erfasst.

Zum Zeitpunkt des Verfassens dieses Beitrags ist die öffentliche und fachliche Diskussion über das ortsunabhängige Arbeiten durch die Erfahrungen der COVID-19 Pandemie und dem im März 2020 in Deutschland (und anderen Ländern) erfolgten Lockdown geprägt. Aus der Notwendigkeit geboren, das Infektionsgeschehen zu minimieren („flatten the curve“), ermöglichten es auch bislang zurückhaltende Unternehmen ihren Mitarbeiter*innen, ihre Tätigkeiten so weit wie möglich von zu Hause aus zu erledigen. Frühe Hochrechnungen ergaben, dass in der ersten Aprilhälfte $202035 \%$ aller Erwerbstätigen ganz oder teileweise zu Hause arbeiteten (Schröder et al. 2020). Analysen des Instituts für Arbeitsmarkt- und Berufsforschung (IAB) zeigten, dass rund jeder zweite Beschäftigte in Betrieben mit mehr als 50 Mitarbeiter*innen zumindest teilweise im Homeoffice arbeitete (Frodermann et al. 2020). Dies macht das Thema unserer Studie Gelingensbedingungen von Homeoffice und mobilem Arbeiten nicht weniger aktuell - im Gegenteil. Denn die Möglichkeit von zu Hause aus zu arbeiten, wurde während des Lockdowns in vielen Fällen zu einer Verpflichtung und dies ungeachtet der konkreten Haushalts- und Arbeitssituation. Fragen danach, ob die häusliche Umgebung gesundes Arbeiten überhaupt ermöglichte, wurden nicht gestellt oder blieben unbeantwortet. Absprachen, etwa der Erreichbarkeit oder der konkreten Arbeitszeiten (und ihrer Erfassung) erfolgten häufig ad hoc oder fehlten ggf. ganz. Dies mag ein Grund dafür sein, dass Untersuchungen zeigen, dass Beschäftigte, die vor der COVID-19 Pandemie keinerlei Erfahrung mit dem Arbeiten von zu Hause hatten, insgesamt etwas unzufriedener mit dem Homeoffice sind als diejenigen, die Homeoffice schon genutzt haben (Bonin et al. 2020).

Für den vorliegenden Beitrag greifen wir auf Befunde aus acht Kurzfallstudien, die wir in kleinen und mittleren Unternehmen (KMU) v.a. in unterschiedlichen Branchen des Dienstleistungssektors zur betrieblichen Re- 
gulierung und Auswirkung von Homeoffice und mobiler Arbeit durchgeführt haben, zurück und analysieren und interpretieren diese Befunde konzeptionell mit Rückgriff auf die Theorie der Zeitgeographie (Hägerstrand 1970). Unsere Ergebnisse zeigen, dass für das Gelingen von Homeoffice und mobiler Arbeit in KMU im Wesentlichen eine gute und verlässliche technische Ausstattung, klare und transparente Regeln und Kommunikation sowie Vertrauen zentral sind.

Der Text ist wie folgt gegliedert: Zunächst werden Begriffsdefinition, Zahlen und Fakten zu ortsflexiblem Arbeiten genannt sowie Vor- und Nachteile von Homeoffice aus betrieblicher und Beschäftigtensicht gegenübergestellt. Danach stellen wir in Abschnitt 3 den zugrundeliegenden konzeptionellen Ansatz der Zeitgeographie anhand von Homeoffice und mobiler Arbeit unter spezieller Berücksichtigung von raum-zeit-institutionellen Zwängen oder Beschränkungen („constraints“) dar. Im Anschluss wird das methodische Vorgehen der Kurzfallstudien (Abschnitt 4) skizziert. In Abschnitt 5 werden anhand der in KMU durchgeführten Kurzfallstudien Möglichkeiten aufgezeigt, den „constraints“ bei der Arbeit im Homeoffice oder an einem flexiblen Ort entgegenzuwirken und somit Gelingensbedingungen für Homeoffice und mobiles Arbeiten aufgezeigt. Abschließend werden die Ergebnisse zusammengefasst und ein Fazit gezogen.

\section{Ortsunabhängiges Arbeiten im Spiegel der Zablen}

Bereits vor den Erfahrungen im Zuge der Corona-Pandemie wurden die Vor- und Nachteile von Homeoffice und mobilem Arbeiten extensiv diskutiert (u.a. Arntz, Yahmed und Belingerieri 2019; Bosua, Kurnia, Gloet und Mendoza 2017; Brenscheidt 2018; Grunau, Ruf, Steffes und Wolter 2019; Lott 2019). Dabei scheinen zumindest in der öffentlichen Wahrnehmung die positiven Seiten des Homeoffice zu überwiegen. Demnach erleichtert das Arbeiten von zu Hause die Vereinbarkeit im weitesten Sinne, ermöglicht eine bessere Vereinbarkeit von privaten und beruflichen Zeitverpflichtungen (auch durch Wegfall der Wegezeiten) oder ein ungestörteres und konzentrierteres Arbeiten. Arbeitgeber*innen können wiederum zum Beispiel von mehr Flexibilität, hoher Motivation und Zufriedenheit der Beschäftigten (zusammenfassend Lott 2020) oder einer geringeren Fluktuation von Mitarbeiter*innen profitieren (Huf 2020).

Weniger häufig wurden die mit dem mobilen Arbeiten verbundenen negativen Aspekte abseits der Fachöffentlichkeit diskutiert, die Erfahrungen während der Pandemie scheinen diese jedoch stärker ins Bewusstsein gerückt zu haben: Entgrenzung, also die Gefahr der Auflösung oder zu- 
mindest des Verschwimmens der Grenzen zwischen Erwerbsarbeit und Privatleben (Gottschall und Voß 2005), die Verletzung von gesetzlicher Arbeits-, Pausen- sowie Ruhezeiten, Auswirkungen auf die Karrierechancen aufgrund fehlender Sichtbarkeit, Vereinsamung durch fehlende soziale Interaktion am Arbeitsplatz und die Tatsache, dass das Arbeiten von zu Hause häufig zu längerer und nicht vergüteter Arbeitszeit führt (Arnold, Steffes und Wolter 2015), aber auch Reibungsverluste, die durch nicht oder nicht vollständig etablierte Kommunikationswege entstehen. Problematisch kann das Homeoffice auch dann werden, wenn es dem Arbeitgeber primär dazu dient, Büroarbeitsplätze durch „Desk-Sharing“ einzusparen und die Beschäftigten kein Mitspracherecht darüber haben, ob ihr Zuhause nun auch Arbeitsort zu sein hat (Herrmann und Frey Cordes 2020). Angaben über die Verbreitung der Möglichkeit, unabhängig vom eigentlichen Arbeitsort zu arbeiten, schwanken deutlich in Abhängigkeit der Quelle (vgl. hierzu Bonin et al. 2020) Neben methodischen Gründen der Datenerhebung spielen hier unterschiedliche Begrifflichkeiten eine Rolle, denn strenggenommen unterscheiden sich mobiles Arbeiten, das Arbeiten im Homeoffice und die Telearbeit voneinander, auch wenn die Begriffe zumindest im Alltagsgebrauch häufig synonym verwendet werden. Für den aktuellen Beitrag lehnen wir uns an die Definition von Kleemann (2005) an und verstehen unter Homeoffice die Möglichkeit, für abhängig Beschäftigte die Arbeit ganz oder zumindest teilweise von zu Hause aus erledigen zu können; dabei ist es für unseren Zugang und die Analyse unerheblich, wie groß der Anteil der Beschäftigten eines Betriebs ist, der Zugang zu dieser Arbeitsform hat oder wie hoch der Anteil der Arbeit ist, die zu Hause erledigt werden kann oder darf (Abendroth und Diewald 2019). Außerdem betrachten wir sowohl formalisierte als auch informelle Regelungen. Mobiles Arbeiten stellt eine „Steigerung“ zu Homeoffice insofern dar, als die Beschäftigten ihre Arbeit auch außerhalb ihrer Wohnung erledigen können. Dagegen zählen wir „mobile Arbeitsformen, die außerhalb des Betriebs ausgeführt werden, weil es die Arbeitstätigkeit verlangt, wie z.B. Kundenbetreuung oder Dienstreisen" (Abendroth und Diewald 2019, S. 82) nicht zu unserem Beobachtungsgegenstand.

Der Anteil der Betriebe in Deutschland, die ihren Mitarbeiter*innen ortsunabhängiges Arbeiten anbieten, ist vor der COVID-19 Pandemie zwar stetig, aber nur langsam gestiegen. Der Anteil lag nach Analysen des für Deutschland repräsentativen IAB-Betriebspanels 2018 bei rund $26 \%$, rechnet man dies auf die Beschäftigtenanzahl hoch, so haben rund $12 \%$ der Beschäftigten Zugang zu einer Form des ortsunabhängigen Arbeitens (Grunau et al. 2019). Als Grund für die doch recht geringfügige Verbreitung des Angebots von Homeoffice führen Personalverantwortliche laut 
Ergebnissen des Linked Personnel Panel (LPP) - das allerdings nur privatwirtschaftliche Betriebe ab 50 Beschäftigte einbezieht und von daher nicht repräsentativ für alle Betriebe ist - vor allem tätigkeitsimmanente Gründe an (90\%). Etwas mehr als jeder Fünfte erwartet Schwierigkeiten in der Zusammenarbeit mit Kolleg*innen (22\%), $16 \%$ haben Datenschutzbedenken, jeder Zehnte ist der Ansicht, Führung und Kontrolle seien im Homeoffice nicht möglich und weitere $10 \%$ geben an, ihre Mitarbeiter"innen hätten kein Interesse. Fehlende technische Voraussetzungen liegen mit $9 \%$ auf dem letzten Platz der angegebenen Gründe (Grunau et al. 2019). Etwas anders fallen die Antworten von Beschäftigten aus, die nicht dezentral arbeiten können: Etwas mehr als drei Viertel der Befragten gibt an, dass ihre Tätigkeit das Arbeiten im Homeoffice nicht zuließe, zwei Drittel stimmen der Aussage zu, dass dem Vorgesetzten die betriebliche Anwesenheit wichtig sei, $59 \%$ befürchten Schwierigkeiten bei der Zusammenarbeit mit Kolleg*innen, $56 \%$ berichten, dass sie die Trennung von Beruf und Privatleben als wichtig erachten und $54 \%$ nennen als Grund mangelnde technische Voraussetzungen. Immerhin $16 \%$ der Befragten führen an, Homeoffice sei, obwohl technisch möglich, nicht erlaubt und $7 \%$ befürchten Karrierehemmnisse (Grunau et al. 2019).

In den Betrieben, in denen dezentrales Arbeiten verbreitet ist, war 2018 nach Auswertungen des IAB-Betriebspanels häufig sowohl das Arbeiten von zu Hause als auch das Arbeiten von unterwegs möglich. In deutlich weniger Betrieben wird hingegen entweder nur Homeoffice oder nur das Arbeiten von unterwegs angeboten (Grunau et al. 2019). Dabei unterscheiden sich die Betriebe bezüglich der Häufigkeit, in der ihre Mitarbeiter"innen zu Hause arbeiten können. Nach Angaben des LPP ist es etwa in einem Sechstel der Betriebe möglich, regelmäßig mindestens einen Tag pro Woche von zu Hause aus zu arbeiten. Weitere $2 \%$ der Betriebe bieten ihren Beschäftigten zwar regelmäßiges Homeoffice an, allerdings weniger als einmal pro Woche und in etwas weniger als jedem fünften Betrieb ist nur unregelmäßiges Homeoffice möglich (Grunau et al. 2019, S. 2). Wie oben beschrieben, bilden zudem betriebliche Charakteristika wie Branche und Größe entscheidende Faktoren, die die Wahrscheinlichkeit des Angebots ortsflexibel zu arbeiten determinieren.

Obwohl das Arbeiten von zu Hause insbesondere die Vereinbarkeit erhöhen soll, arbeiten anteilmäßig Männer häufiger von zu Hause als Frauen (zu den Auswirkungen auf die Kinderbetreuung vgl. Lott 2019). Interessanterweise wird das Homeoffice von Paaren mit Kindern seltener genutzt als von Paaren ohne Kinder (Brenke 2016). Wie bereits ausgeführt, arbeiten zudem Höherqualifizierte und insbesondere Beschäftigte mit Führungsverantwortung häufiger von zu Hause als geringer Qualifizierte und 
Arbeitnehmer*innen ohne Führungsposition (Grunau et al. 2019). Vor diesem Hintergrund sind die Ergebnisse einer aktuellen Analyse von Mergener (2020) interessant, die zeigt, dass der Zugang zu Homeoffice nicht immer rein objektiven Gründen folgt, d.h. tätigkeitsimmanente Ursachen hat. Ob eine bestimmte berufliche Tätigkeit im Homeoffice oder ortsflexibel ausgeübt werden darf, hängt demnach insbesondere von der Betriebsgröße ab (Mergener 2020). Dabei steigt die Wahrscheinlichkeit, Zugang zu Homeoffice zu haben, mit dem Ausmaß an kognitiven Anteilen einer Tätigkeit mit der Betriebsgröße, während die Wahrscheinlichkeit, im Homeoffice arbeiten zu können, für manuelle Tätigkeiten mit steigender Betriebsgröße sinkt. Als Begründung für den ersten Befund dient die Annahme einer besseren technischen und organisationalen Infrastruktur in Großbetrieben mit mehr als 250 Beschäftigten, die den Zugang zu Homeoffice ermöglicht. Dagegen wird die höhere Wahrscheinlichkeit für Zugang zu Homeoffice bei manuellen Tätigkeiten in kleinen Betrieben mit einer familiäreren Betriebskultur begründet, die zu einem höheren Vertrauen in die Beschäftigten führe „somit erlauben sie eher das Durchführen von Tätigkeiten zu Hause, die an sich nicht in hohem Maße dazu geeignet sind“ (Mergener 2020, S. 529).

\section{Konzeptioneller Ansatz der Zeitgeographie}

Grundsätzlich hat Arbeit immer eine zeitliche Dimension, stellt sie doch die Zeit dar, welche Individuen dem Arbeitgeber gegen Entlohnung zur Verfügung stellen. Regelungen zur Arbeitszeit sind daher sowohl Gegenstand umfangreicher staatlicher Regulation als auch stetiges Konfliktthema zwischen den Tarifparteien. Allerdings hat Arbeit auch eine räumliche Dimension, als dass man Arbeitszeit im traditionellen Verständnis als diejenige Zeit verstehen kann, die ein Individuum an der Arbeitsstätte verbringt. Durch solche Zusammenhänge aus Raum und Zeit wird der Alltag von Individuen in modernen, arbeitsteiligen Gesellschaften strukturiert (Giddens 1984). An dieser Stelle beginnt diese Überlegung für das Thema des vorliegenden Aufsatzes relevant zu werden, denn mobile Arbeit oder Homeoffice stellen auch Arbeitszeit dar, allerdings abseits der Arbeitsstätte. Um diesen Zusammenhang schärfer zu analysieren, bietet sich der Rückgriff auf einen konzeptionellen Ansatz an, der die Bedeutung für Raum und Zeit für Individuen in einen gemeinsamen Analyserahmen einbindet. Dies leistet der interdisziplinäre Ansatz der Zeitgeographie (Hägerstrand 1970) dadurch, dass die „Pfade“ („path“) von Individuen in Raum und Zeit in Kontext ihrer physisch und sozialen Handlungsmöglichkeiten sowie Ein- 
schränkungen/Zwänge („net of constraints“) gesetzt werden. Dabei können drei Kategorien von raum-zeit-institutionellen „constraints“ unterschieden werden: „capability constraints“, „coupling constraints“ und „authority constraints".

Unter "capability constraints“ werden körperliche und mentale Eigenschaften (z.B. schlafen, essen, Fähigkeit sich fortzubewegen oder zu kommunizieren) sowie zur Verfügung stehende Mittel (z.B. Werkzeuge, Verkehrsmittel) gefasst, die die Aktivitäten oder „Pfade“ von Individuen beschränken (Hägerstand 1970, S. 12). Dabei gilt naturgemäß, dass ein Individuum zu einer bestimmten Zeit nur an einem bestimmten (physischen) Ort sein kann und Zeit benötigt, um sich von einem Ort zu einem anderen Ort zu bewegen.

In Bezug auf Homeoffice und mobile Arbeit zählen zu den „capability constraints" vor allem die technische Ausstattung und die physische Anwesenheit an einem Ort. Unternehmen, die ihren Mitarbeiter*innen die Möglichkeit anbieten, im Homeoffice oder mobil zu arbeiten, müssen folglich eine technische Infrastruktur bereitstellen sowie das reibungslose Funktionieren und die entsprechende Anpassung dieser auch in Zukunft garantieren. Hierbei sind je nach Bedarf technische Hilfsmittel, wie zum Beispiel Laptops, Tablets, Bildschirme, (Dienst-)Handys, Software und ein sicherer digitaler Datenzugriff auf Arbeitsunterlagen, bspw. durch die Authentifizierung mit Security-Token, notwendig. Die Nutzung digitaler Informations- und Kommunikationstechnologien ist nicht nur für die Durchführung der Arbeitsaufgaben notwendig, sondern dient auch dazu, die Erreichbarkeit technisch zu gewährleisten (die konkrete Ausgestaltung der Erreichbarkeit wird unter "coupling constraints“ ausgeführt). Auch muss sichergestellt werden, dass die technische Ausstattung im Homeoffice oder beim Arbeiten an einem flexiblen Ort den Datenschutzbestimmungen gerecht wird (auch ein „authority constraint“). In einigen Bereichen kann es dabei technisch (noch) nicht anders möglich sein, die Arbeit an einem anderen Ort abseits des Arbeitsplatzes im Unternehmen zu verrichten (z.B. bei großem Datenaustausch oder bei fehlenden finanziellen Mitteln zur technischen Umsetzung). In anderen Bereichen wiederum besteht die Notwendigkeit der physischen Anwesenheit am Arbeitsplatz und der direkten sozialen Interaktion mit Anderen oder der Interaktion mit Materialien und Maschinen, um Tätigkeiten zu verrichten, die „physisch“ nicht in anderer Weise durchgeführt werden können. Dies kann etwa bei der Arbeit mit Patient*innen, bei Handwerkstätigkeiten oder bei der Bedienung einer 
im Betrieb stehenden Maschine ${ }^{1}$ sein. Sofern Arbeitstätigkeiten aus „physischen" und „technischen" Gründen im Homeoffice oder mobil erledigt werden können, entfallen für die Beschäftigten zudem mitunter lange Fahrtwege und die damit einhergehende Zeitersparnis kann die Vereinbarkeit von Privat- und Berufsleben fördern. So kann die Kinderbetreuung oder die Pflege eines Angehörigen leichter mit der Arbeit vereinbart werden, aber auch Erholungszeiten können steigen. Zusätzlich können Umwelt und Klima durch weniger Pendeln geschützt werden (Fischedick und Schneidewind 2020; Hook, Court, Sovacool und Sorrell 2020; Stowasser et al. 2019). Anhand des politischen und öffentlichen Diskurses über innovative und zukunftsfähige Arbeitsmethoden und Arbeitszeitmodelle ${ }^{2}$ sowie über den Klimawandel, kann vermutet werden, dass Homeoffice auch in Zukunft deutlich an Relevanz gewinnen wird. Auch können Arbeitgeber*innen mit Homeoffice auf wetterbedingte (z.B. Schneesturm oder Sturmtief) und gesundheitliche Risiken (z.B. COVID-19 (Bloomberg 2020)) reagieren, um den laufenden Betrieb durch Homeoffice aufrechtzuerhalten.

„Coupling constraints" entstehen aus der Notwendigkeit heraus, dass Individuen sich koordinieren müssen und sich dafür „koppeln“ und „entkoppeln“ müssen („need to couple and de-couple“: Ellegård 2019. S. 44). "Coupling constraints“ bestimmen wo, wann und für wie lange ein Individuum mit einem anderen Individuum, einem Werkzeug oder Material interagiert, um etwas zu produzieren, konsumieren oder durchzuführen (Hägerstand 1970, S. 14). Die räumliche und zeitgleiche Interaktion mit anderen Personen oder Gegenständen ist dabei oftmals sozial oder vertraglich festgelegt. Die Bündelung mehrerer individueller raumzeitlicher „Pfade“ wird dabei als „bundle“ bezeichnet (Hägerstand 1970). In einer Fabrik bilden beispielsweise Maschinen, Materialien und Menschen ein „bundle“, durch das Komponenten verbunden oder getrennt werden. Ein anderes „bundle“ wird etwa im Büro durch technische Hilfsmittel und Menschen gebildet, um Informationen zu verbinden und zu trennen und Nachrichten zu kanalisieren (Hägerstand 1970). Als Sonderform der „bundles“ fasst Hägerstrand die Telekommunikation, bei der nur eine zeitliche, aber keine räumliche Kopräsenz erforderlich ist. Da neuere Informations- und Kommunikationstechnologien die traditionell eher enge Korrespondenz zwi-

1 Wobei bereits auch erste Homeoffice-Möglichkeiten für Fabrikarbeiter*innen getestet werden (Automobilwoche 2019).

2 Zum Beispiel erproben BMAS, Unternehmen und Sozialpartner, wie etwa Gewerkschaften, derzeit neue Arbeitsmodelle in „Experimentierräumen“ (https://ww w.experimentierraeume.de/start/). 
schen Aktivität, Zeit und Ort lockern, und durch virtuelle Kommunikation auch virtuelle Räume geschaffen werden, muss Hägerstrands Ansatz der Zeitgeographie um eine virtuelle Dimension erweitert werden (Melchior, Schiemer und Grabher 2020; Couclelis 2009). So kann durch neuere, internetbasierte Kommunikationsformen die gleiche Aktivität an unterschiedlichen Orten durchgeführt werden (bspw. kann im Unternehmen gearbeitet werden oder an unterschiedlichen Orten) und ein Ort kann zeitweise verschiedene Aktivitäten beherbergen (z.B. im Café mobil arbeiten oder Freizeit im Café verbringen). Durch virtuelle Kommunikation kann zudem der Bedarf, an andere Orte zu reisen, entfallen. Mit den Informations- und Kommunikationstechnologien bzw. virtueller Kommunikation geht somit eine Abschwächung der Assoziation zwischen Zeit und Aktivität einher (z.B. wenn noch spät abends gearbeitet wird). Informations- und Kommunikationstechnologien können somit zu einer Flexibilisierung der Entscheidung führen, wo, wann und wie lange gearbeitet wird.

In einem Arbeitsverhältnis ist die Präsenz am Arbeitsplatz (wo, wann, wie lange) ein zentraler „coupling constraint“. Das „coupling“ von Arbeitszeit und Arbeitsort ist dabei in der Regel fester Bestandteil des Arbeitsvertrages. Da die Arbeitspraktiken in vielen Branchen oder Unternehmensbereichen in hohem Ausmaß durch virtuelle Kommunikation organisiert sind oder mithilfe von Informations- und Kommunikationstechnologien durchgeführt werden, können bestimmte Arbeitstätigkeiten, die keine räumliche und (teils auch keine) zeitliche Kopräsenz erfordern, im Homeoffice und/oder mobil an einem flexiblen Ort durchgeführt werden. Dabei müssen Anwesenheitszeiten im Unternehmen (wo, wann, wie lange) und Zeiten der Erreichbarkeit (in diesem Fall auch das Wie) außerhalb des Unternehmens zwischen einzelnen Individuen ausgehandelt werden. Hierbei muss zudem beachtet werden, dass viele Arbeitsaufgaben zwar aus physischer oder technischer Sicht („capability constraint“) fernab des Unternehmens durchgeführt werden könnten, aus kulturellen Gründen jedoch bevorzugt im direkten persönlichen Austausch im Unternehmen selbst durchgeführt werden. So ist es im Kundendienst oder im Kontakt mit Klientel weiterhin gängig, face-to-face vor Ort zu interagieren. ${ }^{3}$ Aber auch Meetings bzw. räumlich und zeitlich festgelegte Besprechungen zwischen Mitarbeiter*innen werden gewöhnlich im face-to-face Kontakt abgehalten. Handlungen dieser Art können in vielen Fällen zwar auch an einem flexiblen Ort außerhalb des Unternehmens mit technischen Hilfsmitteln, wie

3 Dies weicht allmählich auf, z.B. durch Online-Handel, Online-Banking, OnlineBildungsplattformen, Digitale Stadtverwaltung, etc. 
Telefon- oder Videokonferenz oder im E-Mail-Kontakt (hierbei ohne zeitliche Kopräsenz), durchgeführt werden, oftmals ist es jedoch effizienter und effektiver, diese Aktivitäten physisch und zeitgleich vor Ort durchzuführen, vor allem bei der Teamarbeit, sodass Telekommunikation oder internetbasierte Kommunikation nicht zwangsläufig durch das Einsparen einer räumliche Bewegung zu einer Zeitersparnis oder einem gleichwertigen Ergebnis führen.

"Authority constraints“ begrenzen die Auswahl möglicher Handlungen in bestimmten „domains" (Bereiche oder Gebiete), in denen Dinge und Ereignisse unter der Kontrolle eines bestimmten Individuums oder einer bestimmten Gruppe liegen (Hägerstrand 1970). Handlungen in „domains“ werden durch Macht und informelle oder formelle Institutionen beschränkt. „Domains“ können dabei temporärer (z.B. Warteschlange) oder langfristiger Natur sein und werden zwischen den Individuen ausgehandelt oder sind durch Recht legitimiert (z.B. Zugangsberechtigungen, Eigentumsrechte). Sie sind zudem hierarchisch strukturiert, da sie nur bestimmten Individuen oder Gruppen Zugang gewähren (z.B. Visum oder Aufenthaltsgenehmigung in einem Nationalstaat). So kann ein Unternehmen beispielsweise nur für die Mitarbeiter*innen zugänglich sein, das Vorstandsbüro innerhalb des Unternehmens wiederum nicht für alle Mitarbeiter"innen. Die Kontrolle der "domains“ geht zudem oftmals mit normativen Verhaltenserwartungen an die beteiligten Akteure einher oder es wird erwartet, (formelle und informelle) Regeln zu befolgen (Ellegård 2019). Unternehmensinhaber oder Führungskräfte können zum Beispiel von ihren Mitarbeiter*innen Commitment und Loyalität gegenüber dem Unternehmen erwarten, aber auch innerhalb von Teams wird Commitment gegenüber anderen Teammitgliedern erwartet („demand to be committed", siehe Trygg und Hermelin 2017).

Als ,authority constraints“, speziell in Bezug auf das Arbeiten vor Ort im Betrieb und das Arbeiten im Homeoffice oder mobil, können die zeitliche Reglementierung des Zugangs zum Arbeitsplatz (z.B. Öffnungszeiten), die räumliche Reglementierung des Zugangs (z.B. Fehlen notwendiger Schlüssel) sowie die virtuelle Reglementierung des Zugangs (z.B. fehlender Zugang zu Software- und Clouddiensten) gezählt werden, damit etwa abends oder am Wochenende nicht gearbeitet werden kann. Beim Arbeiten im Homeoffice oder an einem flexiblen Ort außerhalb des Unternehmens müssen zudem gesetzliche Vorschriften, wie etwa Datenschutzbestimmungen, auch im virtuellen Raum eingehalten werden. Auch muss gewährleistet werden, dass gesetzliche Arbeits-, Pausen- und Ruhezeiten eingehalten werden. Neben diesen formellen institutionellen Bestimmungen können auch informelle Rollenerwartungen oder Normen innerhalb 
der „domain" Unternehmen die Arbeit im Homeoffice oder das mobile Arbeiten begrenzen. So herrscht in vielen Unternehmen trotz grundsätzlicher Möglichkeit zu Homeoffice oder mobiler Arbeit eine Präsenzkultur im Betrieb (Lott 2020). Aber auch in Unternehmen, die Homeoffice und mobile Arbeit ermöglichen, wird erwartet, dass ein signifikanter Anteil der Arbeitszeit im Betrieb verrichtet wird, um das reibungslose Funktionieren von Arbeitsabläufen zu ermöglichen und ein gutes Betriebsklima aufrechtzuerhalten.

Zusammenfassend kann an dieser Stelle konstatiert werden, dass eine Implementierung von Homeoffice und mobiler Arbeit der Rücksicht auf alle drei beschriebenen Formen von „constraints“ bedarf. Sowohl physische Präsenszwänge („capability constraints“), die notwendige Abstimmung innerhalb der Organisation beziehungsweise des Teams („coupling constraints") und rechtliche oder organisatorische Zwänge („authority constraints“) müssen aufgelöst bzw. neu adaptiert werden, um ein Gelingen von Homeoffice und mobiler Arbeit zu sichern. Im Folgenden werden mit Hilfe dieser konzeptionellen Sichtweise Lösungsansätze sowie potenzielle Fallstricke empirisch analysiert.

\section{Methodisches Vorgehen: Kurzfallstudien in Betrieben mit Homeoffice und mobiler Arbeit}

Da wir "Gelingensbedingungen“ untersuchen wollten, konzentrierten wir uns bei der methodischen Auswahl unserer Betriebe auf Betriebe mit "guter Praxis“. Das Auswahlkriterium „gute“ betriebliche Praxis von Homeoffice und mobiler Arbeit stellt zunächst eine Herausforderung dar, steht die Bewertung einer Praxis als „gute Praxis“ in der Regel am Ende des Forschungs- und Evaluationsprozesses. Um eine Vorauswahl zu treffen, wurden Unternehmen und Betriebe kontaktiert, die als familienfreundlich zertifiziert worden sind („audit berufundfamilie“, „Qualitätssiegel familienfreundlicher Arbeitgeber“, „Erfolgsfaktor Familie“) und Homeoffice und/ oder mobile Arbeit bereits eingeführt haben. Dabei stellte der Betrieb die Untersuchungseinheit dar. Hierbei ist es gelungen, Kurzfallstudien in acht Betrieben verschiedener Branchen durchzuführen (siehe Tabelle 1; siehe auch Bonin et al. 2020). Die acht Betriebe weisen eine Beschäftigtenzahl von 46 (Softwaredienstleister) bis 490 (Textilverarbeitung) auf, und das Durchschnittsalter der Beschäftigten beträgt 40 Jahre oder höher. Der Frauenanteil liegt bei den meisten untersuchten Unternehmen (mit Ausnahme des Softwaredienstleisters und des Ingenieurbüros) bei über $50 \%$, und in allen Betrieben existiert die Möglichkeit, in unterschiedlichen For- 
men Teilzeit zu arbeiten. Außerdem weist die Hälfte der untersuchten Unternehmen eine Form der Beschäftigtenvertretung auf.

Die Kurzfallstudien basieren vorwiegend auf leitfadengestützten Interviews, die mit Personalverantwortlichen geführt wurden, in zwei Fällen mit der Geschäftsführung und in einem Fall auch zusätzlich mit dem Betriebsrat. Die Dauer der leitfadengestützten Interviews variierte zwischen 45 und 90 Minuten. Die Interviews wurden digital aufgezeichnet, im Sinne der Fragestellung zusammengefasst und inhaltsanalytisch ausgewertet. Darüber hinaus wurden in einigen Fällen betriebsinterne Dokumente wie Betriebsvereinbarungen und FAQs zur Verfügung gestellt oder es wurde Einsicht in mobile Apps und Computerprogramme, etwa zur Zeiterfassung oder Statusmeldung, gegeben. 
Flexibel in Zeit und Raum

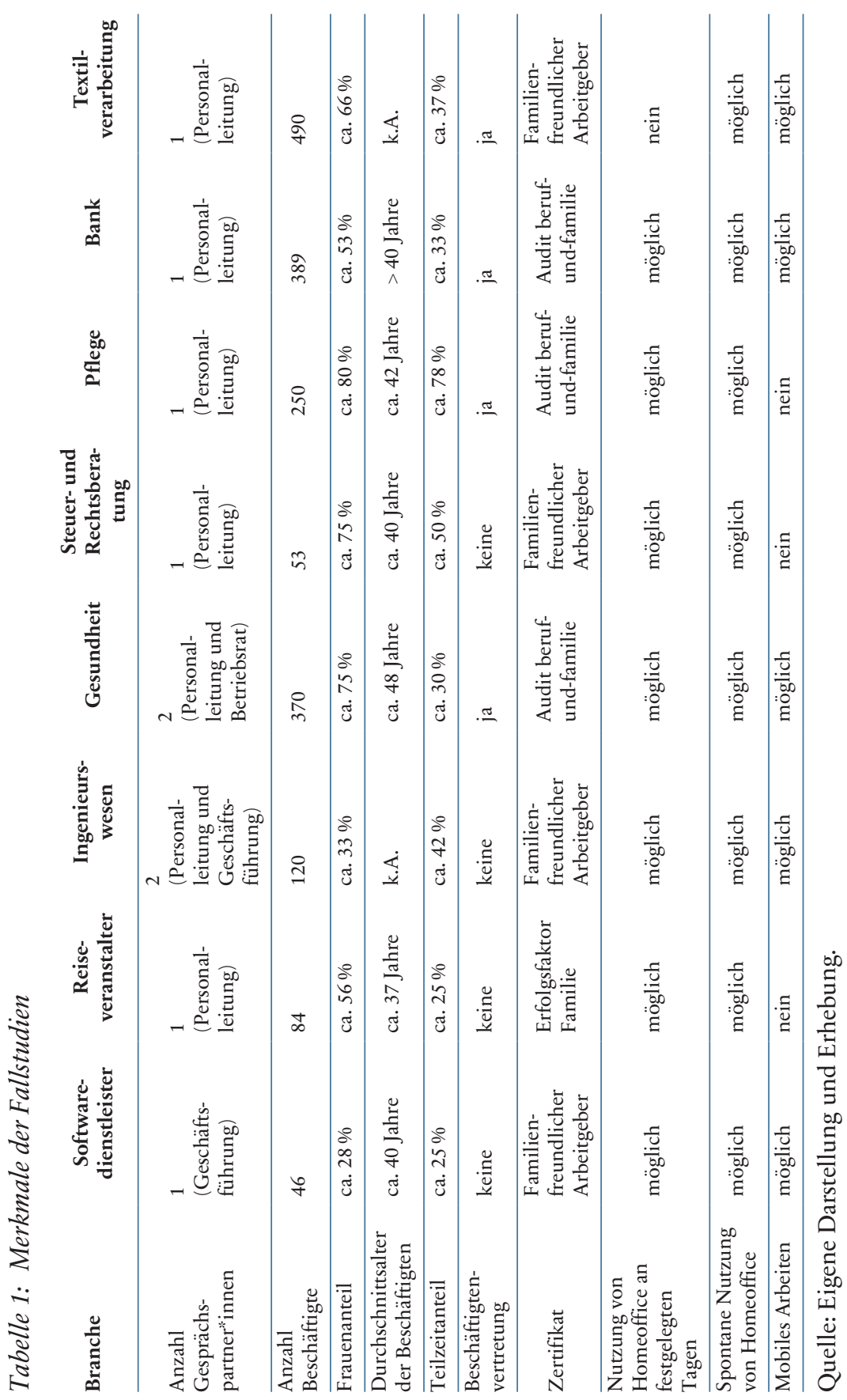




\section{Ausgestaltung von Homeoffice und mobiler Arbeit in der Praxis von KMU}

Die acht untersuchten Fallbetriebe weisen bei der Nutzung und Ausgestaltung von Homeoffice und mobiler Arbeit viele Gemeinsamkeiten, aber auch Unterschiede auf. In sieben der acht Betriebe ist eine Nutzung von Homeoffice an vorher festgelegten und in der Regel nicht veränderbaren Tagen möglich (bei allen außer Textilverarbeitung, siehe Tabelle 1). Eine spontane, situationsbedingte Nutzung von Homeoffice ist in allen acht Betrieben möglich. In fünf der acht Betriebe (Softwaredienstleister, Ingenieurswesen, Gesundheit, Bank und Textilverarbeitung) kann auch mobil, d.h. ortsflexibel gearbeitet werden.

Nicht alle Beschäftigtengruppen haben in der Regel gleichermaßen Zugang zu der Möglichkeit, mobil oder im Homeoffice zu arbeiten, diese Unterschiede sind vor allem aber nicht nur auf die Art der Tätigkeit zurückzuführen. Während bei Ingenieurswesen die Hälfte der Beschäftigten sehr flexibel und mobil arbeitet, da ein spezifisches Arbeitscharakteristikum darin besteht, regelmäßig zu Baustellen und externen Terminen mit Planungspartnern zu fahren, kann die Arbeit am Empfang hingegen nicht mobil oder im Homeoffice durchgeführt werden. Kennzeichnend für einen Pflegeanbieter wiederum ist die Arbeit an und mit Patient*innen direkt vor Ort im Betrieb, insofern beschränkt sich die Möglichkeit des Homeoffice bei Pflege auf Führungskräfte, die vor allem mit administrativen Tätigkeiten beschäftigt sind. Sie sind mit mobilen Tablets und Smartphones ausgestattet und nutzen ein aktuelles Pflegeverwaltungsprogramm. Im Betrieb Gesundheit besteht hingegen ein Großteil der Tätigkeit in der Erstellung von Gutachten und administrativen Tätigkeit, ein Großteil der Beschäftigten mit Gutachtertätigkeit arbeitet daher mobil und von zu Hause aus.

Das Arbeiten im Homeoffice, in einigen Fällen auch die mobile Arbeit, ermöglicht es den Beschäftigten, Fahrtwege einzusparen, insbesondere wenn der Wohnsitz mehr als eine Stunde vom Betrieb entfernt ist. Bei Reiseveranstalter arbeiten beispielsweise zwei Mitarbeiterinnen an drei Tagen vor Ort im Betrieb und die übrigen zwei Tage im Homeoffice. Auch bei Ingenieurswesen arbeitet eine Person, die in deutlicher Entfernung zum Betrieb wohnt, an vier von fünf Tagen im Homeoffice. Bei Softwaredienstleister haben einige Mitarbeiter*innen beantragt, dauerhaft im Homeoffice zu arbeiten, um Wegzeiten und Fahrtkosten zu sparen und arbeiten nun durchweg von zu Hause aus.

Je nach Arbeitsbereich und individueller Arbeitstätigkeit können nach Aussagen des Managements Mitarbeiter*innen im Homeoffice oder an einem flexiblen Ort bei der Durchführung ihrer Arbeit auf verschiedene Herausforderungen oder Beschränkungen („,constraints“) stoßen. Die 
Kurzfallstudien zeigen, dass für das Gelingen von Homeoffice und mobiler Arbeit vor allem drei Bedingungen erfüllt sein müssen. Ist die Art der individuellen Arbeitsaufgaben grundsätzlich für das Arbeiten im Homeoffice oder an einem anderen Ort außerhalb des Betriebs möglich, müssen Mitarbeiter"innen angemessen technisch ausgestattet sein, um ihre Arbeitsaufgaben erledigen zu können („capability constraint“). Außerdem müssen zwischen Beschäftigten und Geschäftsführung, direkten Vorgesetzten und Teammitgliedern klare und transparente Absprachen und Regeln der Anund Abwesenheit sowie Erreichbarkeit getroffen werden („coupling constraint“). Auch sollte eine Kultur des Vertrauens praktiziert werden (,authority constraint“).

\subsection{Technische Ausstattung}

Die Durchführung von Arbeitsaufgaben und die (technische) Erreichbarkeit an einem flexiblen Ort wird grundsätzlich durch die Nutzung digitaler Informations- und Kommunikationstechnologien ermöglicht. Für Homeoffice oder mobile Arbeit ist je nach Art der Tätigkeit eine Grundausstattung an IT-Hard- und Software, sowie ein internetbasierter Zugriff auf das Firmennetz und benötigte Dateien, unerlässlich. Letzteres kann auch durch onlinebasierte Speicher- und Serverdienste (Clouds) und automatische Versionskontrolle von Dateien vereinfacht werden. Projektmanagementsoftwares (z.B. Trello oder Asana) und Kommunikationssoftwares (z.B. Slack, Microsoft Teams, Google Hangouts, Zoom) ermöglichen wiederum die Koordination von Arbeitsaufgaben und den (orts- und teils auch zeitunabhängigen) Kontakt zwischen Beschäftigten.

Bei Softwaredienstleister erhalten alle im Homeoffice oder mobil arbeitenden Mitarbeiter*innen auf Wunsch die gleichen Geräte gestellt, die sie auch im Büro nutzen (u.a. Laptop, zwei Bildschirme). Zur technischen Ausstattung muss auch die Qualität der Daten- oder Serververbindung gezählt werden, die vor allem für Betriebe mit hohem Datenaustausch relevant sind (Softwaredienstleister, Ingenieurswesen). Dabei kann es vorkommen, dass die Qualität der Datenverbindung im Büro deutlich besser ist als zu Hause oder an einem anderen Ort, sodass die Arbeit im Betrieb effizienter erledigt werden kann (Softwaredienstleister).

Einige der untersuchten Fallbetriebe sind laut Aussagen unser Gesprächspartner*innen technische Vorreiter in ihrer Branche (Softwaredienstleister, Steuer-und Rechtsberatung) und trieben die Digitalisierung und damit einhergehend auch die Einführung von Homeoffice und mobiler Arbeit im Branchenvergleich bereits vergleichsweise früh voran. Auch ar- 
beiten einige der Fallstudienbetriebe bereits fast vollständig ohne Papier und haben ihre Akten digitalisiert (Steuer- und Rechtsberatung, Bank, Gesundheit), im Zuge dessen müssen bei der Arbeit im Homeoffice oder an einem flexiblen Ort keine Arbeitsunterlagen mitgenommen werden. Bei Softwaredienstleister nutzen alle Beschäftigten zudem eine App, die ihren Status (abwesend, nicht stören, verfügbar, etc.) anzeigt, bei Textilverarbeitung tragen Mitarbeiter"innen ihre Erreichbarkeit in einem Arbeitskalender ein, der für alle einsehbar ist. Auf diese Weise sind alle Teammitglieder über die Erreichbarkeit ihrer Kolleg*innen informiert.

In einigen der untersuchten Betriebe liegen jedoch Unterschiede bei der technischen Ausstattung der Mitarbeiter"innen vor (Bank, Gesundheit), sodass es für einige Beschäftigtengruppen, wie z.B. den Verwaltungsangestellten bei Gesundheit, entweder noch überhaupt nicht möglich ist, im Homeoffice oder mobil zu arbeiten, oder es müssen die entsprechenden Geräte ausgeliehen werden, wobei dafür vorgesehene Anmeldefristen eingehalten werden müssen. Auch wenn noch nicht alle Mitarbeiter*innen mit eigenen Geräten ausgestattet sind, betonen viele Interviewpartner*innen, dass die weitere Digitalisierung vorangetrieben wird und sich Unterschiede in der Ausstattung zukünftig vermindern werden. In diesem $\mathrm{Zu}$ sammenhang ist der finanzielle Aspekt bei der technischen Ausstattung von Beschäftigten im Homeoffice oder in mobiler Arbeit nicht zu vernachlässigen. Auch stellt jeder Homeoffice-Arbeitsplatz für die IT-Stelle einen gesonderten Arbeitsplatz dar, der nach Aussage der Personalleitung von Gesundheit "gepflegt“" werden müsse, d.h. Software auf den neusten Stand gebracht, was wiederum einen Kostenfaktor darstellt. Zusätzlich werden in einigen der untersuchten Betriebe die heimischen Arbeitsplätze bei ständiger oder regelmäßiger Homeoffice-Tätigkeit mindestens stichprobenartig geprüft (z.B. bei Reiseveranstalter, Bank).

Ein weiterer wichtiger Aspekt der technischen Ausstattung von Beschäftigten bei der Arbeit im Homeoffice oder an einem flexiblen Ort besteht hinsichtlich des Umgangs mit persönlichen Daten und der Einhaltung von Datenschutzbestimmungen. So werden die Datenschutzbestimmungen bei Bank technisch durch Passwörter und die Nutzung eines persönlichen Sicherheits-Tokens gewährleistet. Durch die persönliche Identifikation per Sicherheits-Token wird es den Beschäftigten ermöglicht, zu Hause oder an einem Ort ihrer Wahl zu arbeiten. Bei Steuer- und Rechtsberatung hingegen ist das mobile Arbeiten in öffentlichen Räumen nicht gestattet, da der Datenschutz in diesem Fall, im Gegensatz zu einer sicheren Verbindung von zu Hause oder aus nichtöffentlichen Räumen (z.B. in einem Hotelzimmer), noch nicht vollkommen garantiert werden kann. Für die Anmeldung an den Geräten wird ein entsprechender Sicherheitsstick verwendet. 
Zusätzlich wird der Datenschutz im Homeoffice gewährleistet, indem man die Mandant"innen zu Beginn auch eine Einverständniserklärung unterschreiben lässt (Datenschutzbestimmungen sind hierbei auch ein „authority constraint"). Ähnliches gilt auch bei Gesundheit, wo hochsensible medizinische Daten verarbeitet werden.

Als einen weiteren relevanten Aspekt für die Inanspruchnahme von Homeoffice oder mobiler Arbeit, der sich eher auf die individuellen Fähigkeiten der einzelnen Mitarbeiter*innen bezieht, nannten einige Interviewpartner*innen die Fähigkeit zur Selbstorganisation oder Selbstdisziplin. In einigen der Fallbetriebe wurde den Beschäftigten hierfür die Teilnahme an Kursen zum Zeit- und Selbstmanagement angeboten (Gesundheit, Steuerund Rechtsberatung). Auch die Nutzung von Projektmanagement-Software kann Zeitmanagement fördern (und kann dabei gleichzeitig auch „coupling constraints" lösen).

\subsection{Klare und transparente Regeln und Kommunikation}

Für die betriebliche (Zusammen-) Arbeit ist es von fundamentaler Bedeutung, dass die Beschäftigten sich mit Vorgesetzten und Kolleg*innen koordinieren und miteinander im Austausch stehen. Die Interviewpartner*innen berichten, dass mit der Nutzung von Homeoffice und/oder mobiler Arbeit grundsätzlich vor allem ein erhöhter Kommunikations- und Koordinationsaufwand einhergehe. Daher müssen Anwesenheitszeiten (wo, wann, wie lange) und Zeiten der Erreichbarkeit (wo, wann, wie lange, wie) bei der Arbeit außerhalb des Betriebs klar und transparent abgestimmt werden. Hierfür konnten in den untersuchten Unternehmen unterschiedliche Regelungsarten beobachtet werden, um der Entwicklung des erhöhten Abstimmungsbedarfs ein Stück weit entgegenzuwirken. So gibt es im Hinblick auf die Inanspruchnahme von Homeoffice und mobiler Arbeit in einigen der untersuchten Unternehmen einheitliche Regeln für alle Beschäftigten oder zumindest gleiche Regeln für bestimmte Beschäftigtengruppen (z.B. Betriebsvereinbarung bei Textilverarbeitung), während andere wiederum auf individuell vertragliche Lösungen für Homeoffice/mobiles Arbeiten setzen. Ob in den jeweiligen Unternehmen hierbei gleiche Regeln für alle oder individuelle Lösungen gefunden werden, ergibt sich unter anderem aus der Art der jeweiligen Arbeitstätigkeiten, hängt mit der Unternehmenskultur und dem Führungsstil zusammen, aber auch mit der Existenz und Rolle von kollektiven Interessensvertretungsstrukturen. 
Auch können die Beschäftigten entweder regelmäßiges oder spontanes Homeoffice in Anspruch nehmen. In der Regel wird Homeoffice ganztägig in Anspruch genommen. Einige Beschäftigte haben dabei auf eigenen Wunsch feste Anwesenheitstage im Betrieb und feste Homeoffice-Tage festgelegt (Reiseveranstalter, Steuer- und Rechtsberatung). Bei Pflege müssen die festen und regelmäßigen Homeoffice-Tage beantragt und vertraglich festgeschrieben werden. Es kann aber auch vorkommen, dass Homeoffice nur stundenweise genutzt wird, teilweise auch „on top“ abseits der regulären Arbeitszeit, um etwa noch eine Arbeitsaufgabe in einer bestimmten Frist fertigzustellen (Pflege, Steuer- und Rechtsberatung).

Bei Ingenieurswesen können die Beschäftigten beispielsweise selbstständig entscheiden, wann sie kommen und gehen. Auch können private Termine, wie etwa Arztbesuche, eingeschoben werden, sofern es die Projektund Terminplanung zulässt (auch bei Softwaredienstleister möglich). In einigen der Fallunternehmen werden regelmäßige Anwesenheitstage (wöchentlich, monatlich oder auch quartalsweise) festgelegt, an die sich alle beteiligten Mitarbeiter*innen halten sollen, bei Softwaredienstleister gelten feste Anwesenheitstage etwa für eine spezielle Gruppe der Entwickler*innen. In vielen Fällen sind hierbei vor allem die Unternehmens- oder Teamleitung gefragt, klare und transparente Regeln aufzustellen, die auch in schriftlicher Form etwa im Intranet (Reiseveranstalter), Unternehmenswiki oder FAQ (Softwaredienstleister) für alle einsehbar sind.

Hingegen wurde in anderen der untersuchten Unternehmen jedoch bewusst auf das Aufstellen zu vieler Regeln für Homeoffice und mobile Arbeit verzichtet, um ein hohes $\mathrm{Ma}$ an Flexibilität zu garantieren (Ingenieurswesen, Textilverarbeitung) oder weil die Einführung des Homeoffice, wie die Personalleitung aus Steuer- und Rechtsberatung es formulierte, als „schleichender Prozess“ erfolgte und sich eine Praxis auch ohne formale Regeln etabliert hat. In den Fallunternehmen ohne starke Regulierung, aber mit einem großen Anteil an Teamarbeit, treffen die Teammitglieder daher untereinander verbindliche Absprachen (Ingenieursunternehmen, Textilverarbeitung). In den Fallunternehmen, in der die Arbeit sehr selbstständig organisiert wird, gelten lediglich Erreichbarkeitsregeln oder, wie bei Steuer- und Rechtsberatung, die Regel, dass $40 \%$ der Arbeit im Unternehmen selbst zu erledigen ist (dazu mehr in Abschnitt 5.3).

Bei allen untersuchten Betrieben gilt außerdem der Grundsatz, dass betriebliche Belange Vorrang vor Homeoffice und mobiler Arbeit haben und die Anwesenheit vor Ort, etwa bei Besprechungen, in der Regel erwartet wird. Daher können Anfragen im Homeoffice zu arbeiten auch temporär abgelehnt werden (Ingenieurswesen). Hierbei kann vor allem für Mitarbeiter*innen, die in viele Schnittstellen einbezogen sind, der Handlungsspiel- 
raum in der Gestaltung der Arbeitszeit und somit auch in der Nutzung von Homeoffice begrenzt sein (Textilverarbeitung). Dies und die unterschiedliche Freiheit, die zum Teil in den Teams herrscht, kann auch potentiell zu Konflikten führen. Aber auch das ist Teil der Unternehmenskultur bei Textilverarbeitung.

Wie stark sich Beschäftigte mit anderen Beschäftigten koordinieren müssen, hängt von der Tätigkeit und Arbeitsorganisation ab. Ist die Arbeitsaufgabe eines Beschäftigten grundsätzlich eher individuell und selbstständig durchführbar, kann die Arbeit mit weniger Koordinationsaufwand außerhalb des Betriebs verrichtet werden. Das trifft beispielsweise auf Rechercheaufgaben oder das Rechnungswesen zu, wie z.B. bei Reiseveranstalter, oder auf individuelle Bearbeitung von Fällen oder Gutachten, wie z.B. bei Steuer- und Rechtsberatung und Gesundheit. Ist die Arbeit zum Großteil hingegen eher teamförmig organisiert, nimmt die Bedeutung von Absprachen, Erreichbarkeit und Teilnahme an Meetings zu. Vor allem bei Softwaredienstleister und Ingenieurswesen arbeitet ein Großteil der Beschäftigten in Teams. Daher werden Termine auch möglichst rechtzeitig festgelegt. In Fällen, in denen eigentlich die Anwesenheit vor Ort gefragt wäre, können Mitarbeiter*innen in einigen der untersuchten Unternehmen bei Bedarf jedoch auch per Telefon- oder Videokonferenz zugeschaltet werden (Ingenieurswesen).

Grundsätzlich gilt in allen untersuchten Betrieben, dass Beschäftigte, die mobil oder im Homeoffice arbeiten, erreichbar sein müssen. Die Erreichbarkeit kann telefonisch oder online (etwa per Mail) gewährleistet werden (Textilverarbeitung). Beschäftigte, die ortsflexibel arbeiten, sind angehalten, Erreichbarkeit auch dadurch herzustellen, dass sie die Weiterleitungsfunktion ihres Firmentelefons aktivieren und Anrufe auf das eigene Festnetz oder Handy weiterleiten lassen (Textilverarbeitung). Aber auch die Nutzung von Kommunikationssoftware kann die Koordination von Aufgaben und den Austausch zwischen Mitarbeiter*innen sicherstellen.

Für die Frage, wann und wie lange Beschäftigte bei Homeoffice und mobiler Arbeit erreichbar sein sollen, ist vor allem auch die konkrete Ausgestaltung der Arbeitszeit von Bedeutung. So sollen die Beschäftigten etwa während der Kernarbeitszeit oder Funktionsarbeitszeit auch im Homeoffice erreichbar sein (z.B. Softwaredienstleister 9-16 Uhr bzw. 7 bis $18 \mathrm{Uhr}$ ), vor allem bei der Funktionsarbeitszeit sind die Beschäftigten bzw. Teams selbst dafür verantwortlich, die Erreichbarkeit, auch gegenüber Kundin*innen, während dieser Zeit sicherstellen. In einigen Fällen kann hierbei festgestellt werden - auch wenn die Erreichbarkeit im Homeoffice nicht ausdrücklich geregelt ist - doch die Erwartung besteht, zumindest während der Bürozeiten ( 8 bis $16 \mathrm{Uhr}$ ) erreichbar zu sein (Pflege). In Unternehmen 
mit hohem Kundenkontakt kommt hinzu, dass nicht nur die unternehmensinterne Erreichbarkeit bei Homeoffice oder mobiler Arbeit gewährleistet sein muss, sondern auch die unternehmensexterne Erreichbarkeit, beispielsweise während der (Kern-) Arbeitszeiten. Bei Bank wird aus diesem Grund erwartet, dass die Mitarbeiter"innen aufgrund der hohen Kunden- und Serviceorientierung des Unternehmens grundsätzlich im Homeoffice erreichbar sind und Anfragen per E-Mail zeitnah innerhalb eines Arbeitstags und während der Servicezeiten beantworten. Auch werden bei mobiler Arbeit Absprachen über Zuständigkeit und Erreichbarkeit innerhalb des Teams getroffen.

\subsection{Vertrauen, unterschiedliche Präferenzen und Teamkultur}

Neben der technischen Ausstattung und den klaren Regeln und Absprachen wurde in sieben von acht befragten Betrieben gegenseitiges Vertrauen als weiterer wesentlicher Punkt für das Gelingen von Homeoffice und mobiler Arbeit genannt. Hierbei wurde auch darauf hingewiesen, dass Vertrauen erlernt werden könne und in einigen Fällen auch erst erlernt werden musste (Softwaredienstleister). Ein Gesprächspartner der Geschäftsführung von Softwaredienstleister berichtete in diesem Zusammenhang durchaus auch selbstkritisch, dass es ihm anfangs nicht leichtfiel, allen Mitarbeiter*innen das entsprechende Vertrauen im gleichen Ausmaß entgegenzubringen „das musste erst gelernt werden“. Diese Einschätzung wird auch von anderen Gesprächspartner*innen geteilt, denn auch bei Bank standen einige Führungskräfte der Ausweitung von mobilem Arbeiten auf die breite Belegschaft anfangs skeptisch gegenüber. Bei Bank wurde dieses Vertrauen per „doing“ gelernt. Dies war so überzeugend, dass mobiles Arbeiten mittlerweile in allen Abteilungen nicht nur akzeptiert, sondern auch geschätzt wird.

Die Nutzung von Homeoffice hängt jedoch nicht nur von objektiven Kriterien wie Tätigkeitszuschnitten, technischer Ausstattung oder Wünschen der Beschäftigten ab, sondern es spielen auch Kulturfragen eine Rolle. So gibt es in vielen Betrieben eine unterschiedliche Nutzung von Homeoffice über die Abteilungen hinweg. Nach Ansicht der Personalleitung von Textilverarbeitung sind es auch gewachsene Teamkulturen, die die Nutzung von Homeoffice bestimmen. Nach Einschätzung eines Betriebsrats bei Gesundheit, müssen in Teams, in denen von Homeoffice bisher wenig Gebrauch gemacht wurde, erst noch positive Erfahrungen gesammelt werden. Die Gesprächspartnerinnen von Pflege und Textilverarbeitung machen zusätzlich zu unterschiedlichen Kulturen in den Teams auch unterschiedli- 
che Präferenzen jüngerer und älter Beschäftigten aus. Während ältere Beschäftigte eine starke Präsenzorientierung besäßen, würde die Möglichkeit (rotierend) mobil oder im Homeoffice zu arbeiten von den jüngeren Mitarbeiter*innen mittlerweile erwartet und stelle auch keinen besonderen Wettbewerbsvorteil mehr hinsichtlich des Wettbewerbs auf dem Arbeitsmarkt um neue Beschäftigte dar.

Gegenseitiges Vertrauen, ein gutes Betriebsklima oder eine bestimmte Art von Teamkultur sind jedoch keine Eigenschaften, die als gegeben angenommen werden können oder keiner Pflege bedürften. Vor diesem Hintergrund betonen einige Gesprächspartner*innen explizit die Wichtigkeit einer regelmäßigen physischen Anwesenheit am Arbeitsplatz im Betrieb für effektives Arbeiten, Ideenaustausch und Teambindung (Softwaredienstleister, Ingenieurswesen, Steuer- und Rechtsberatung).

Deshalb gibt es in allen von uns untersuchten Fällen Vereinbarungen, die eine gemeinsame Anwesenheit zu bestimmten Zeiten zumindest fördern: Softwaredienstleister verpflichtet z.B. alle Entwickler*innen zu einem festen Anwesenheitstag und auch alle Teamleitungen müssen mindestens einen Arbeitstag pro Woche im Büro verbringen. Der Informationsfluss wird dadurch sichergestellt, dass alle Teams täglich zeitlich feststehende Runden haben, in denen sich ausgetauscht wird und bei denen die Anwesenheit Pflicht ist. Wobei Anwesenheit auch durch Kommunikationssoftwares wie Skype hergestellt werden kann. Zudem nutzen alle Beschäftigten, auch diejenigen, die vor Ort arbeiten, eine Software, die ihren Status anzeigt (abwesend, nicht stören, verfügbar etc.), damit alle Teammitglieder informiert sind, wen sie wann ansprechen können. Einmal im Quartal treffen sich die Teams zudem zu Feedbackgesprächen, in denen angesprochen wird, was gut, was weniger gut gelaufen ist und wie zufrieden die Beschäftigten aktuell sind. Ähnliche regelmäßige Treffen, die dem persönlichen Austausch dienen und in denen nur notfalls virtuell teilgenommen werden kann und sollte, gibt es auch im Ingenieurbüro, bei Gesundheit und im Pflegedienst. Bank sowie Steuer- und Rechtsberatung haben dagegen in ihren Regelungen vereinbart, dass der Großteil der Arbeitszeit vor Ort im Büro zu leisten ist. Vor diesem Hintergrund wird auch deutlich, warum eine Vielzahl unserer Gesprächspartner*innen explizit unterstrichen hat, dass das Angebot des ortsflexiblen Arbeitens nicht zu einer Reduzierung der Büroarbeitsplätze genutzt werden solle. Ortsflexibles Arbeiten stellte zumindest bei unseren Befragten - ein Angebot an die Beschäftigten dar, um die (subjektiven) Arbeitsbedingungen zu verbessern und für das Unternehmen bessere Resultate zu erzielen - aber kein Mittel der Kosteneinsparung. 


\section{Fazit}

Ziel des Beitrags war zu zeigen, welche Bedingungen gegeben sein müssen, damit Homeoffice und/ oder mobiles Arbeiten auch in KMU, in denen diese Arbeitsformen bisher unterrepräsentiert sind, funktionieren kann. Hierfür wurden Kurzfallstudien in acht Betrieben unterschiedlicher Branchenzugehörigkeit und Größe durchgeführt, die Beispiele guter Praxis darstellen sollten. Um dies zu gewährleisten, haben wir uns bei der Fallauswahl auf als familienfreundlich zertifizierte Betriebe konzentriert. Dabei sind wir davon ausgegangen, dass ortsflexibles Arbeiten aufgrund unterschiedlicher Faktoren auf Hindernisse stoßen kann, die wir mit Rückgriff auf den Ansatz der Zeitgeographie in drei Kategorien („capability constraints“, „coupling constraints“ und „authority constraints“) eingeteilt haben.

Unsere Studie zeigt, welche Maßnahmen und Praktiken KMU etabliert haben, um solchen Beschränkungen entgegenzuwirken. Dabei ist ein zentrales Ergebnis, dass die befragten Betriebe jeweils spezifische Lösungen, die die spezifische Arbeitskultur widerspiegelt, für das mobile Arbeiten oder das Arbeiten im Homeoffice gefunden haben. $\mathrm{Zu}$ den wesentlichen allgemeinen Bedingungen des Gelingens für Homeoffice und mobile Arbeit zählen hierbei vor allem die technische Ausstattung, eine klare und transparente Regelung von Arbeitsprozessen und Kommunikation sowie eine Kultur des Vertrauens und Teamkultur im Betrieb.

Die praktischen Erfahrungen der Fallbetriebe belegen deutlich die branchenübergreifende Eignung von Homeoffice und mobilem Arbeiten auch in KMU bei adäquater Implementierung. Darüber hinaus muss sich ortsflexibles Arbeiten auch nicht auf bestimmte berufliche Positionen beschränken. Zentral für die betriebliche Entscheidung, das zeigen unsere Fallbetriebe in Einklang mit den Ergebnissen quantitativer Studien, Homeoffice oder mobiles Arbeiten anzubieten, sind die Tätigkeitszuschnitte der Beschäftigten, insbesondere inwieweit die Beschäftigten Kundenkontakt haben oder - wie etwa in der Pflege - ihre Tätigkeit direkt am Menschen erfolgt. Aber auch in diesen Fällen zeigen sich in den Fallstudien häufig Lösungsmöglichkeiten - etwa, wenn Teilleistungen der Arbeit mobil oder im Homeoffice erbracht werden können. Möglich wird dies unter anderem auch durch die Verknüpfung von mobilem Arbeiten oder Homeoffice mit flexiblen Arbeitszeiten und/oder Funktionszeiten. Das Team regelt dann die Anwesenheit während der Öffnungszeiten unter sich und ermöglicht so einzelnen Teammitgliedern ganztägiges oder stundenweises mobiles Arbeiten. Alle befragten Arbeitgeber und der Betriebsrat waren mit ihren Regelungen sehr zufrieden, unabhängig von ihrer Komplexität 
oder der Regelungstiefe. Viele Unternehmen planen daher eine Ausweitung auf weitere Beschäftigte.

Ein weiteres wichtiges Ergebnis der Fallstudien ist aber auch, dass die regelmäßige Anwesenheit und Kommunikation vor Ort und innerhalb der Abteilungen oder Teams seitens des Managements und dem Betriebsrat als essentiell eingeschätzt wird. Entsprechend ist in den Betrieben oftmals geregelt, dass ein bestimmter Anteil, häufig sogar der Hauptanteil der Arbeitszeit, vor Ort erbracht werden muss. Andere Unternehmen haben regelmäßige Termine festgelegt, an denen sich sämtliche Mitarbeiter"innen vor Ort zu Team- oder Abteilungstreffen einzufinden haben. In allen untersuchten Fallbetrieben gilt dabei das Prinzip, dass betriebliche Belange Vorrang haben und dass Arbeiten fern des Betriebs nur möglich ist, solange es nicht zu signifikanten Nachteilen in Bezug auf die (Zusammen-)Arbeit kommt.

Vor dem Hintergrund, dass der Gesetzgeber aktuell eine Regelung zu gesetzlichen Rahmen und Ansprüchen an Homeoffice anstrebt, ist eines unserer Ergebnisse als besonders wichtig hervorzuheben, nämlich, dass es einer genauen Passung zwischen den Tätigkeitszuschnitten, den Anforderungen des Betriebes und nicht zuletzt den Bedürfnissen der Beschäftigten bedarf. Was in einem bestimmten Betrieb gut funktioniert, muss nicht automatisch auch für andere Betriebe gelten. An dieser Stelle wollen wir auch noch einmal unterstreichen, dass es sich bei der Auswahl der untersuchten Betriebe um eine Positivauswahl handelte, also um Betriebe, bei denen Themen wie die Work-Life Balance ihrer Mitarbeiter*innen hoch auf der Agenda stehen, was die Übertragbarkeit unserer Ergebnisse auf andere Betriebe einschränken kann. Ungeachtet dessen zeigten sich die meisten unserer Gesprächspartner"innen optimistisch, dass sich das von ihnen gelebte Modell flexiblen Arbeitens auch auf andere Betriebe oder Unternehmen übertragen ließe, verweisen aber auch in den Gesprächen häufig auf spezifische Arbeitskulturen, die sich bei ihnen entwickelt hätten. Dass immer mehr Unternehmen, vor allem ausgelöst durch die COVID-19 Pandemie, ihren Mitarbeiter*innen Homeoffice zugänglich machen und auch weiterhin ermöglichen wollen, regelmäßig im Homeoffice zu arbeiten, verdeutlicht umso mehr, dass Homeoffice oder ortsflexibles Arbeiten zukünftig in der Arbeitswelt an Relevanz gewinnen wird. Ein klärender gesetzlicher Rahmen für das Arbeiten im Homeoffice kann dabei als Katalysator für die Einführung von Homeoffice wirken. Bei der konkreten Ausgestaltung spezifischer betrieblicher Vereinbarungen und Praktiken sollten Management und Beschäftigte bzw. betriebliche Interessenvertretungen verschiedene Faktoren (wie Ausstattung, Absprachen und Vertrauen) be- 
rücksichtigen, damit Homeoffice nicht nur realisiert wird, sondern auch für alle Seiten gelingt.

\section{Literatur}

Abendroth, A.-K., \& Diewald, M. (2019). Auswirkungen von Teleheimarbeit auf geschlechtsspezifische Einkommensungleichheiten in Arbeitsorganisationen. Die Bedeutung unterschiedlicher Umsetzungsformen. Kölner Zeitschrift für Soziologie und Sozialpsychologie, 71(1), 81-109.

Arnold, D., Steffes, S., \& Wolter, S. (2015). Mobiles und entgrenztes Arbeiten. BMAS Forschungsbericht 460. Verfügbar unter: http://www.bmas.de/SharedDo cs/Downloads/DE/PDF-Publikationen/Forschungsberichte/f460-mobiles-und-en tgrenztes-arbeiten.pdf\%3F_blob\%3DpublicationFile\%26v\%3D1 [23.09.2020].

Arntz, M., Yahmed, B., \& Berlingieri, F. (2019). Working from home: Heterogeneous effects on hours worked and wages. ZEW Discussion Paper, Nr.19-015: Mannheim: ZEW. Verfügbar unter: http://ftp.zew.de/pub/zew-docs/dp/dp19015.pdf [01.10.2020].

Automobilwoche (2019). Elektronikfertigung im Wohnzimmer: Conti testet offenbar Homeoffice für Fabrikarbeiter. Verfügbar unter: https://www.automobilwoc he.de/article/20190610/NACHRICHTEN/190619998/elektronikfertigung-im-wo hnzimmer-conti-testet-offenbar-homeoffice-fuer-fabrikarbeiter [18.06.2020].

Bloomberg (2020). Coronavirus forces world's largest work-from-home experiment. https://www.bloomberg.com/news/articles/2020-02-02/coronavirus-forcesworld-s-largest-work-from-home-experiment https:/www.bloomberg.com/news/ articles/2020-02-02/coronavirus-forces-world-s-largest-work-from-home-experiment[18.06.2020].

Bonin, H., Eichhorst, W., Kaczynska, J., Kümmerling, A., Rinne, U., Scholten, A., \& Steffes, S. (2020). Verbreitung und Auswirkungen von mobiler Arbeit und Homeoffice. Kurzexpertise im Auftrag des BMAS. Verfügbar unter: https://www .bmas.de/SharedDocs/Downloads/DE/PDF-Publikationen/Forschungsberichte/f b-549-pdf-verbreitung-auswirkung-mobiles-arbeiten.pdf;jsessionid $=820401300 \mathrm{~B}$ C4E5B82A87A2A83F7F188E.delivery2-master?__blob=publicationFile\&v=1 [11.10.2020].

Bosua, R., Kurnia, S., Gloet, M., \& Mendoza, A. (2017). Telework Impact on Productivity and Well-Being. In J. Choudire, S. Kurnia \& P. Tsatsou (Hrsg.), Social Inclusion and Usability of ICT-Enabled Services (S. 201-223). New York: Routledge.

Brenke, K. (2016). Home Office: Möglichkeiten werden bei weitem nicht ausgeschöpft. DIW Wochenbericht Nr. 5/2016 (S. 95-106). Berlin: DIW.

Brenscheidt, F. (2018). Arbeitszeit 4.0 - Orts- und zeitflexibles Arbeiten: Gesundheitliche Chancen und Risiken. Dortmund: Bundesanstalt für Arbeitsschutz und Arbeitsmedizin. 
Couclelis, H. (2009). Rethinking time geography in the information age. Environment and Planning A, 41(7), 1556-1575.

Ellegård, K. (2019). Thinking time geography: Concepts, methods and applications. London: Routledge.

Eurostat (2020). Employed persons working from home as a percentage of the total employment, by sex, age and professional status. Verfügbar unter: https://appsso .eurostat.ec.europa.eu/nui/submitViewTableAction.do [01.10.2020].

Fischedick, M., \& Schneidewind, U. (2020). Folgen der Corona-Krise und Klimaschutz - Langfristige Zukunftsgestaltung im Blick behalten. Diskussionspapier. Wuppertal: Wuppertal Institut für Klima, Umwelt, Energie. Verfügbar unter: https://wupperinst.org/fa/redaktion/downloads/publications/Corona-Krise_Klim aschutz.pdf [01.10.2020].

Frodermann, C., Grunau, P., Haepp, T., Mackeben, J., Ruf, K., Steffes, S., \& Wanger, S. (2020). Online-Befragung von Beschäftigten: Wie Corona den Arbeitsalltag verändert hat. IAB-Kurzbericht, 13/2020. Verfügbar unter: http://doku.iab.de /kurzber/2020/kb1320.pdf [23.09.2020].

Giddens, A. (1984). The constitution of society: Outline of the theory of structuration. Berkeley: University of California Press.

Gottschall, K., \& Voss, G.G. (2005). Entgrenzung von Arbeit und Leben: Zum Wandel der Beziehung von Erwerbstätigkeit und Privatsphäre im Alltag (2. Auflage). München und Mering: Rainer Hampp Verlag.

Grunau, P., Ruf, K., Steffes, S., \& Wolter, S. (2019). Mobile Arbeitsformen aus Sicht von Betrieben und Beschäftigten: Homeoffice bietet Vorteile, hat aber auch Tücken. IAB-Kurzbericht, 11/2019. Verfügbar unter: http://doku.iab.de/kur zber/2019/kb1119.pdf [18.06.2020].

Hägerstrand, T. (1970). What about people in Regional Science? Regional Science Association, 24(1), 6-21.

Herrmann, M., \& Frey Cordes, R. (2020). Homeoffice im Zeichen der Pandemie: Neue Perspektiven für Wissenschaft und Praxis? IUBH Discussion Papers - Human Resources, No. 2/2020, Erfurt: IUBH Internationale Hochschule.

Hook, A., Court, V., Sovacool, B., \& Sorrell, S. (2020). A systematic review of the energy and climate impacts of teleworking. Environmental Research Letters, 15(9), 093003.

Huf, S. (2020). Personalmanagement. Wiesbaden: Springer Gabler.

Kleemann, F. (2005). Die Wirklichkeit der Teleheimarbeit. Eine arbeitssoziologische Untersuchung. Berlin: edition sigma.

Lott, Y. (2020). Work-Life Balance im Homeoffice: Was kann der Betrieb tun. WSI Report, Nr. 54. Düsseldorf: Hans-Böckler-Stiftung. Verfügbar unter: https://ww w.boeckler.de/pdf/p_wsi_report_54_2020.pdf [23.09.2020].

Lott, Y. (2019). Weniger Arbeit mehr Freizeit? WSI Report, Nr. 47. Düsseldorf: Hans-Böckler-Stiftung. Verfügbar unter: https://www.boeckler.de/pdf/p_wsi_rep ort_47_2019.pdf [23.09.2020]. 
Melchior, A., Schiemer, B., \& Grabher, G. (2020). „Hägerstrand online “: Ein methodisches Konzept zur Analyse raumzeitlicher Trajektorien in Kollaborationen. Raumforschung und Raumordnung Spatial Research and Planning, 78(1), 2133.

Mergener, A. (2020). Berufliche Zugänge zum Homeoffice. Ein tätigkeitsbasierter Ansatz zur Erklärung von Chancenungleichheit beim Homeofficezugang. Kölner Zeitschrift für Soziologie und Sozialpsychologie, 72(2), 1-24.

Schröder, C., Entringer, T., Göbel, J., Grabka, M., Graeber, D., Kröger, H., Kroh, M., Kühne, S., Liebig, S., Schupp, J., Seebauer, J., \& Zinn; S. (2020). Vor dem Covid-19-Virus sind nicht alle Erwerbstätigen gleich. DIW-Aktuell Nr. 41. Berlin: DIW. Verfügbar unter: https://www.diw.de/de/diw_01.c.789505.de/publikat ionen/diw_aktuell/2020_0041/vor_dem_covid-19-virus_sind_nicht_alle_erwerbs taetigen_gleich.html [18.06.2020].

Stowasser, S., Altun, U., Hartmann, V., Hille, S., \& Sandrock, S. (2019). Gutachten zur mobilen Arbeit. Verfügbar unter: https://www.arbeitswissenschaft.net/filead min/Downloads/Angebote_und_Produkte/Publikationen/FDP_Gutachten_Mob ile_Arbeit_Finale_Version_15.10.2020.pdf [11.10.2020].

Trygg, K., \& Hermelin, B. (2017). Work practice among advanced producer service firms-project work in space-time. Geografisk Tidsskrift-Danish Journal of Geography, 117(1), 11-21. 